University of Massachusetts Amherst

ScholarWorks@UMass Amherst

2005

\title{
Enhanced fixation reveals the apical cortical fringe of actin filaments as a consistent feature of the pollen tube
}

\author{
A Lovy-Wheeler \\ KL Wilsen \\ TI Baskin \\ PK Hepler
}

Follow this and additional works at: https://scholarworks.umass.edu/biology_faculty_pubs

Part of the Biology Commons

\section{Recommended Citation}

Lovy-Wheeler, A; Wilsen, KL; Baskin, Tl; and Hepler, PK, "Enhanced fixation reveals the apical cortical fringe of actin filaments as a consistent feature of the pollen tube" (2005). Planta. 24.

https://doi.org/10.1007/s00425-004-1423-2

This Article is brought to you for free and open access by the Biology at ScholarWorks@UMass Amherst. It has been accepted for inclusion in Biology Department Faculty Publication Series by an authorized administrator of ScholarWorks@UMass Amherst. For more information, please contact scholarworks@library.umass.edu. 


\section{Alenka Lovy-Wheeler · Kathleen L. Wilsen \\ Tobias I. Baskin · Peter K. Hepler \\ Enhanced fixation reveals the apical cortical fringe of actin filaments as a consistent feature of the pollen tube}

Received: 13 September 2004

\begin{abstract}
The actin cytoskeleton plays a crucial role in the growth and polarity of the pollen tube. Due to inconsistencies in the conventional preservation methods, we lack a unified view of the organization of actin microfilaments, especially in the apical domain, where tip growth occurs. In an attempt to improve fixation methods, we have developed a rapid freeze-whole mount procedure, in which growing pollen tubes (primarily lily) are frozen in liquid propane at $-180^{\circ} \mathrm{C}$, substituted at $-80^{\circ} \mathrm{C}$ in acetone containing glutaraldehyde, rehydrated, quenched with sodium borohydride, and probed with antibodies. Confocal microscopy reveals a distinct organization of actin in the apical domain that consists of a dense cortical fringe or collar of microfilaments starting about $1-5 \mu \mathrm{m}$ behind the extreme apex and extending basally for an additional $5-10 \mu \mathrm{m}$. In the shank of the pollen tube, basal to the fringe, actin forms abundant longitudinal filaments that are evenly dispersed throughout the cytoplasm. We have also developed an improved ambient-temperature chemical fixation procedure, modified from a protocol based on simultaneous fixation and phalloidin staining. We removed EGTA, elevated the $\mathrm{pH}$ to 9, and augmented the fixative with ethylene glycol bis[sulfosuccinimidylsuccinate] (sulfo-EGS). Notably, this protocol preserves the actin cytoskeleton in a pattern similar to that produced by cryofixation. These procedures provide a reproducible way to preserve the actin cytoskeleton; employing them, we find that a cortical fringe in the apex and finely dispersed longitudinal filaments in the shank are consistent features of the actin cytoskeleton.
\end{abstract}

Keywords Actin - Actin preservation · Pollen tube · Chemical fixation $\cdot$ Cryofixation $\cdot$ Sulfo-EGS

A. Lovy-Wheeler · K. L. Wilsen · T. I. Baskin · P. K. Hepler ( $ه)$ Department of Biology and Plant Biology Graduate Program, Morrill Science Center III, University of Massachusetts, 611 North Pleasant St., Amherst, MA, 01003-9297, USA E-mail: hepler@bio.umass.edu

Tel.: + 1-413-5452083

Fax: $+1-413-5453243$

\section{Introduction}

Pollen tubes extend rapidly in a polar fashion, searching for the ovule, to deliver the sperm cells to the embryo sac and thus complete fertilization. Among the many components that play a central role in pollen tube growth is the actin cytoskeleton. It has long been appreciated that actin microfilaments, together with myosin, generate cytoplasmic streaming, and thus provide the mechanism that transports the vesicles, which contain cell-wall precursors, to the tip where they fuse and participate in cell elongation. More recently, evidence has emerged that actin polymerization also participates in pollen tube growth. Several agents, including latrunculin, cytochalasin, DNAse and profilin, block growth at concentrations significantly lower than those needed to inhibit cytoplasmic streaming (Gibbon et al. 1999; Vidali et al. 2001), supporting the idea that actin polymerization is essential for growth.

Although actin activity in the apical domain plays a central role in pollen tube growth, we currently lack a comprehensive and consistent view of the structure and organization of actin microfilaments in this region of the growing cell. In the shank of the pollen tube, virtually all studies show longitudinally oriented cables of actin. By contrast, in the apex there is no consensus, despite numerous attempts to resolve this issue (Condeelis 1974; Perdue and Parthasarathy 1985; Pierson et al. 1986; Pierson 1988; Tiwari and Polito 1988; Tang et al. 1989; Heslop-Harrison and Heslop-Harrison 1991). In most studies, actin has been localized by chemical fixation at room temperature, followed by staining with phalloidin or anti-actin antibodies. However, the pollen tube, perhaps because of its rapid, oscillating growth rate (Holdaway-Clarke and Hepler 2003), or considerable turgor pressure, has proved difficult to fix in a life-like state. As a consequence, during conventional fixation, there is often extensive and disruptive rearrangement of internal structure, including notably actin microfilaments (He and Wetzstein 1995; Doris and Steer 1996). 
A survey of published reports indicates that several different actin organizations were observed. In images of pear and tobacco pollen tubes, dense accumulations of actin extend to the extreme apex of the tube (Tiwari and Polito 1988; Tang et al. 1989; Derksen et al. 1995), while in other studies of the pollen tubes, the extreme apex is depleted in F-actin ( $\mathrm{Li}$ et al. 2001; Raudaskoski et al. 2001; Vidali et al. 2001). A few published micrographs of lily, tobacco, and maize pollen tubes have shown a fringe or collar of F-actin consisting of short, longitudinal microfilaments extending to within $1-5 \mu \mathrm{m}$ of the pollen tube tip (Pierson et al. 1986; Yokota et al. 1995; Kost et al. 1998; Gibbon et al. 1999), but this configuration has not been observed commonly. Finally, two advances in chemical fixation have generated yet different views of apical actin organization. Firstly, pretreatment with maleimidobenzoyl hydroxysuccinimide (MBS) to stop cytoplasmic streaming before fixation produces a subapical domain that contains a funnel-shaped actin structure and an apex that contains sparse filaments (Doris and Steer 1996; Vidali et al. 2001). Secondly, the application of simultaneous fixation with aldehydes and staining with phalloidin yields images, in which the entire apical and subapical domains are filled with a meshwork of random short actin microfilaments (Foissner et al. 2002).

Another approach to imaging actin has involved rapid freeze fixation, which, in contrast to the slow reaction times characteristic of ambient-temperature chemical fixation, stabilizes cytoplasmic structures in only a few milliseconds. When the apical domain of cryofixed, freeze-substituted tobacco and lily pollen tubes was examined at the electron microscope level, relatively few actin microfilaments were observed (Lancelle and Hepler 1989; Lancelle and Hepler 1991; Miller et al. 1996). However, while cryofixation presumably preserves actin filaments reliably, a finely dispersed array of microfilaments may escape detection due to a lack of contrast.

As an alternative to any fixation, actin microfilaments were localized in living pollen tubes with fluorescently tagged proteins, but here too, a consensus on actin organization has yet to emerge. Using GFP-talin as an actin marker in tobacco pollen tubes, one study reported randomly arranged, short actin fragments in the apical domain (Fu et al. 2001), whereas another reported a ring of bright fluorescence around the apical organelle exclusion zone (Kost et al. 1998). More recently, actin depolymerizing factor (ADF) fused to GFP, which was employed as an actin probe in both tobacco and lily pollen tubes, reveals a fluorescent band a few microns behind the apex (Chen et al. 2002). This band, which is referred to as a mesh of actin rather than a fringe, appears to be expressed throughout the thickness of the tube, as indicated by confocal microscopy. Beyond the issue of actin organization, it must also be noted that live cell probes have not adequately resolved microfilaments, especially in the apex.
Because the structure of actin in the apical region of the pollen tube remains unresolved, we have revisited the issue in hopes of developing techniques that will reveal apical-actin structures reproducibly and reliably. The basis of our approach was to combine the exceptional structural preservation offered by cryofixation with the high-contrast labeling of fluorescence microscopy. Cryofixation is customarily the province of electron microscopy, but the enhanced preservation it provides is readily apparent through the light microscope (Baskin et al. 1996). Fluorescence microscopy, although unable to resolve detailed spatial arrangements, nevertheless allows us to survey the disposition of the whole actin array rapidly. This latter task has also been facilitated by the development of methods to handle the pollen tube as a whole mount, without requiring embedment or sectioning.

With this approach, we have developed a protocol for the reproducible staining of pollen tubes. We have focused on pollen tubes of two species of lily, and to a lesser extent on tobacco. We also developed a markedly improved ambient-temperature chemical fixation method, which yields results that are similar to those produced by cryofixation. The conclusion from this study is that growing pollen tubes, especially those of lily, consistently show a dense cortical fringe or collar of actin microfilaments in the apical domain. Given its position, it seems plausible that this fringe represents a fragile but rapidly turning over actin array that establishes a close association with the plasma membrane, and that might play a pivotal role in defining growth rate and polarity.

\section{Materials and methods}

\section{Cell culture}

Lilium formosanum plants were first grown from seed in growth chambers, then transferred to the greenhouse. $L$. longiflorum plants were grown in the greenhouse from bulbs (kindly provided by the Gloeckner Company). Nicotiana tabacum plants were grown under standard greenhouse conditions. Pollen was used fresh or frozen $\left(-80^{\circ} \mathrm{C}\right)$, and grown in a medium containing $15 \mathrm{mM}$ MES, $1.6 \mathrm{mM} \mathrm{BO}_{3} \mathrm{H}, 1 \mathrm{mM} \mathrm{KCl}, 0.1 \mathrm{mM} \mathrm{CaCl}_{2}, 7 \%$ sucrose, $\mathrm{pH}$ 5.5. For plunge freezing, the level of sucrose was raised to $10 \%$.

\section{Rapid freeze fixation and immunolabeling}

Lilium longiflorum and $N$. tabacum pollen was surfacegerminated on pollen tube growth medium for 2-4 h, picked up on 3\% agar loops, and plunge frozen at $-180^{\circ} \mathrm{C}$ in liquid propane and freeze-substituted at $-80^{\circ} \mathrm{C}$ for $36 \mathrm{~h}$ in dry acetone as previously described (Lancelle et al. 1986). The freeze-substitution medium was supplemented with $2-5 \%$ anhydrous glutaraldehyde 
and after $36 \mathrm{~h}$, samples were brought to room temperature over a period of $8 \mathrm{~h}$. The loops containing the pollen tubes were rehydrated in 10\% steps (15-30 min each) to $100 \%$ water, and then placed into $10 \mathrm{mM}$ phosphate buffered saline (PBS), a modified method from Fields et al. (1997). Pollen tubes were treated with $0.1 \% \mathrm{NaBH}_{4}$ for $15 \mathrm{~min}$, then rinsed with PBS supplemented $0.1 \%(\mathrm{w} / \mathrm{v})$ Tween-20 for $15 \mathrm{~min}$. A monoclonal mouse anti-actin antibody (C4-raised against chicken gizzard actin, Chemicon, Temecular, CA, USA), or a mouse monoclonal anti-alpha-tubulin (raised against sea urchin axonemes, Sigma, St. Louis, MO, USA), was applied at $4^{\circ} \mathrm{C}$ overnight, washed six times in PBS supplemented with $0.05 \%$ Tween-20, and secondary antibody (Cy-3-goat anti-mouse, Jackson ImmunoResearch Laboratories, Inc., West Grove, PA, USA) was applied for $3-4 \mathrm{~h}$ at $37^{\circ} \mathrm{C}$. Pollen tubes were washed six times with PBS supplemented with $0.05 \%$ Tween 20 for 5 min each, and mounted in 4\% $n$-propyl gallate $(90 \%$ glycerol, $10 \%$ PBS).

For embedding and sectioning, rapidly frozen cells were frozen as described above, freeze-substituted in acetone at $-80^{\circ} \mathrm{C}$ for $36 \mathrm{~h}$, and slowly brought to room temperature (over a period of 8-10 h). Pollen tubes on loops were then infiltrated with butyl methyl methacrylate (BMM) using ethanol as a transitional solvent $(25 \%$ steps). When infiltrated with 75\% BMM: $25 \%$ ethanol, vials were left in the hood overnight, allowing most of the ethanol to evaporate. Pollen tubes were then infiltrated in fresh $100 \%$ resin, allowed to sit for $2 \mathrm{~h}$, and this step was repeated two more times. Samples were polymerized in flat-bottom BEEM capsules under UV light overnight. Sections $(2 \mu \mathrm{m})$ were cut with glass knives, and immunolabeling was performed as reported (Baskin et al. 1996). Imaging was performed on a Zeiss LSM510 Meta confocal microscope using the HeNe $543 \mathrm{~nm}$ laser excitation, and a long pass 568 emission filter.

\section{Chemical fixation}

Lilium formosanum, L. longiflorum, or N. tabacum pollen tubes were cultured $1-2 \mathrm{~h}$ and simultaneously fixed and permeabilized with a buffer composed of $100 \mathrm{mM}$ PIPES (or $100 \mathrm{mM}$ TAPS), $5 \mathrm{mM}$ MgSO4, $0.5 \mathrm{mM}$ $\mathrm{CaCl} 2,0.05 \%$ Triton $\mathrm{X}-100,1.5 \%$ formaldehyde, and $0.05 \%$ glutaraldehyde at $\mathrm{pH} 9$ for $0.5-1 \mathrm{~h}$. Ethylene glycol bis[sulfosuccinimidylsuccinate] (sulfo-EGS) was added at $5 \mathrm{mM}$, and $N$-(maleimidobenzoyloxy)-succinimide (MBS) at $0.1 \mathrm{mM}$. Sulfo-EGS was also used at $0.1 \mathrm{mM}$, but $5 \mathrm{mM}$ gave better results. MBS precipitated at $5 \mathrm{mM}$. The growth medium was completely removed before adding the fixative. The fixative was then washed out two times with the same buffer as above except at pH 7 with $10 \mathrm{mM}$ EGTA, and $6.6 \mu \mathrm{M}(10 \mu \mathrm{l} /$ ml) Alexa 543-phalloidin (Molecular Probes). Images could be taken as early as $15 \mathrm{~min}$, but the best results were obtained $1-2 \mathrm{~h}$ after staining. Confocal imaging was performed as described above for cryofixation.

\section{Results}

Confocal imaging of rapidly frozen whole mounts

Pollen tubes were plunge-frozen in liquid propane, freeze-substituted in acetone containing glutaraldehyde, warmed, re-hydrated in phosphate buffered saline (PBS), quenched with sodium borohydride, and then incubated with antibodies. Examination of these whole mounts with the confocal microscope reveals clear labeling of the actin cytoskeleton; in Fig. 1, five replicate lily pollen tubes are shown.

As a test of the quality of the whole mounts, we examined the structure of the pollen tubes using DIC optics (Fig. 1a-e). The plunge-frozen cells have well preserved clear zones and resemble living cells. Fluorescence microscopy of these cells reveals a distinct fringe of actin in the apical domain (Fig. 1f-o). Beginning about $1-5 \mu \mathrm{m}$ back from the extreme apex, a sharp transition occurs from virtually no signal to one that is bright. This signal consists of closely packed, longitudinally oriented microfilaments that extend basally for approximately an additional $5-10 \mu \mathrm{m}$. Out of 44 cells examined, 37 exhibited a clear fringe. Of the seven that did not show a distinct fringe, four appeared to be poorly fixed and had only faint labeling in the apical region, possibly due to lack of penetration of the antibody, and one was very short possibly indicating poor growth. The variable distance where the fringe first appears $(1-5 \mu \mathrm{m})$ may reflect the particular phase of oscillatory growth during which the pollen tube was frozen. Optical sectioning with the confocal microscope reveals that the filaments making the fringe reside in the cortical cytoplasm, immediately adjacent to the plasma membrane (Fig. 1f-j). Images from medial optical sections show that a non-stained region occurs in the cytoplasm in the area of the fringe, extending for 10 $15 \mu \mathrm{m}$ from the tip, and corresponding in outline to the vesicle rich "inverted cone" (Lancelle and Hepler 1992).

The microfilaments in the shank are also longitudinally oriented, but they are less densely packed than those of the apical fringe (Fig. 2). There is a rather distinct transition from the fringe to the shank, denoted by a diminution in the numbers of microfilaments. Even so, the two arrays appear to be closely related insofar as some filaments in the shank appear to originate in the fringe. Some of these microfilaments curve inward from the fringe towards the central core of the pollen tube, creating a funnel-shaped configuration (Fig. 1f $-\mathrm{j}$ ). As with the cortical fringe itself, the depth of the funnel varies among individuals, again possibly depending on the phase of oscillatory growth during which the pollen tube was frozen. Pollen tubes from tobacco exhibited similar actin labeling (Fig. 3) to those from lily. Thus, a cortical fringe or collar of actin is seen in the apical domain, which begins about $1-3 \mu \mathrm{m}$ from the apex and extends for roughly another $3-5 \mu \mathrm{m}$. 
Fig. 1 Apical actin organization in plunge frozen, rehydrated, and immunolabeled lily pollen tubes. a-e DIC images of five healthy pollen tubes; $\mathbf{f}-\mathbf{j}$ single confocal medial optical sections; $\mathbf{k}-\mathbf{o}$ whole cell projections. These images reveal a prominent cortical actin fringe that is composed of longitudinally oriented actin microfilaments. $\operatorname{Bar}=10 \mu \mathrm{m}$
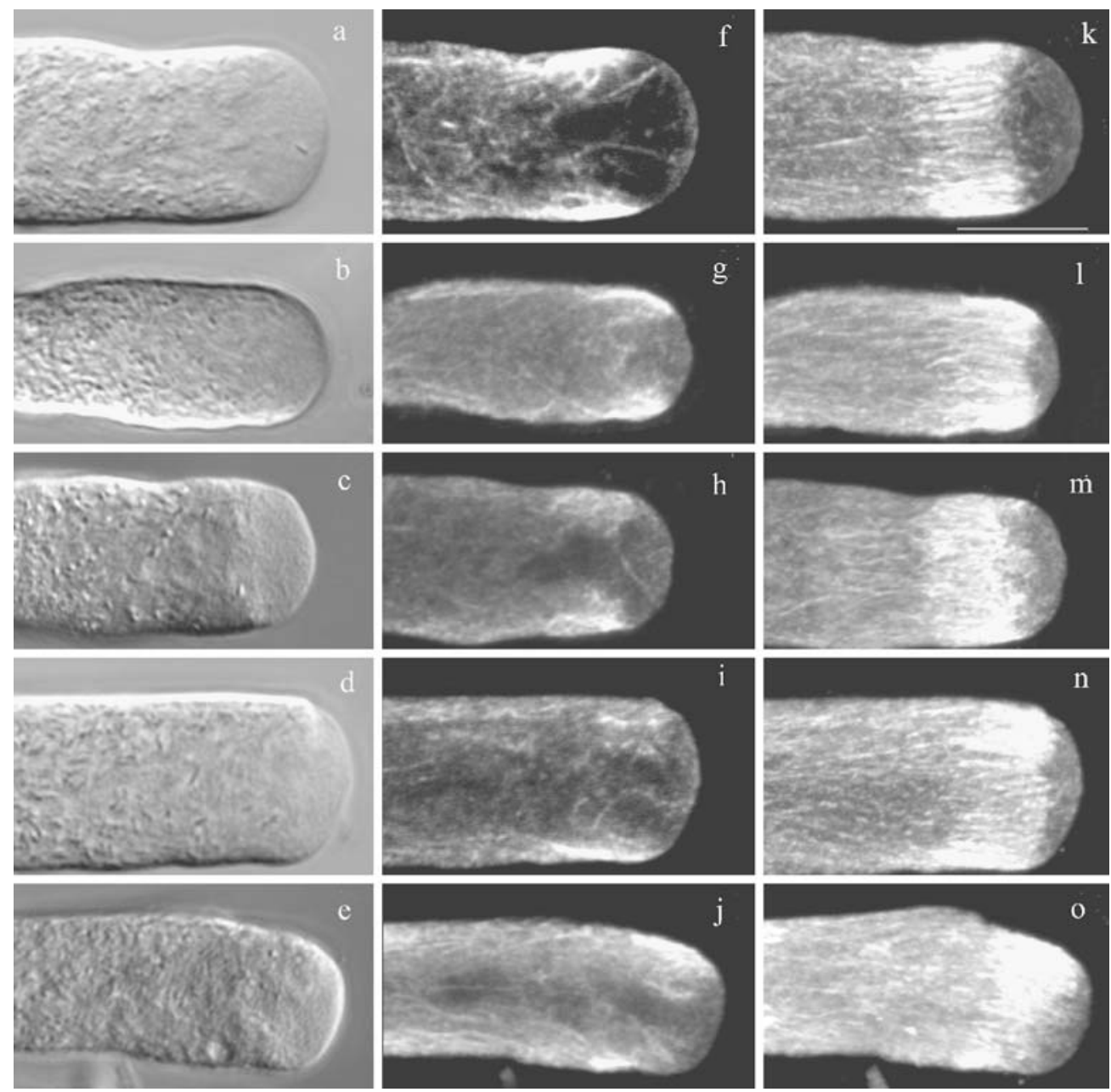

Fig. 2 Actin organization in plunge-frozen, rehydrated, and immunolabeled lily pollen tubes. a, b Shanks of the same tubes shown in Fig. 1k, n. Projection images. Bar $=10 \mu \mathrm{m}$. Note the evenly dispersed actin microfilaments throughout the shank
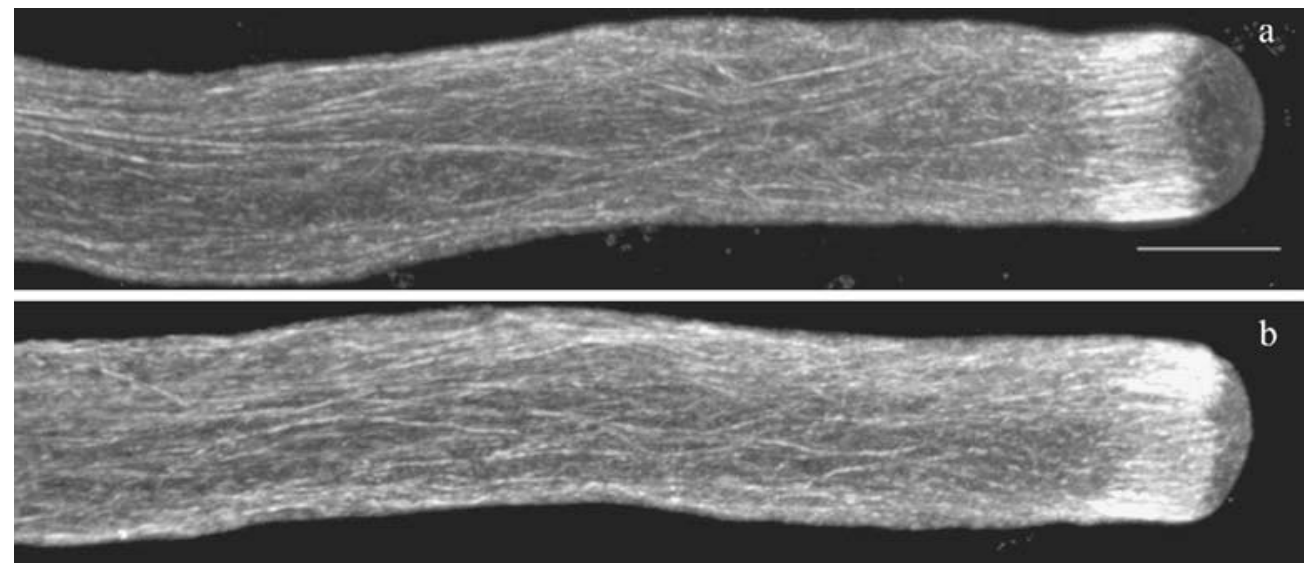

For this study, we also used cryofixation, followed by freeze-substitution without glutaraldehyde, embedment in butyl-methyl-methacrylate (BMM), and sectioning. We thought that images from these sections would provide high detail, and further that the images from successive sections could be reconstructed to produce the three-dimensional pattern. Additionally, since this procedure does not involve any cross-linking reagents, we could examine possible artifacts arising from these chemicals. Indeed, this procedure confirms the structure and organization of the fringe (Fig. 4a, b). However, reconstructing the global organization of the microfilaments proved difficult. Invariably some sections, particularly those from the tangential surfaces, are missing, making it nearly impossible to render a meaningful whole.

To test the whole mount procedure further, we probed lily pollen tubes with antibodies against other antigens for 


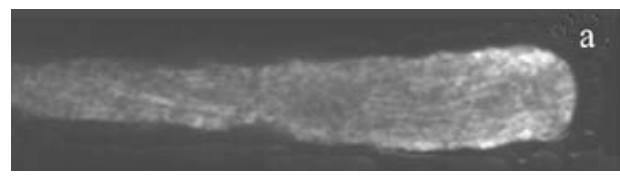

Fig. 3 Apical-actin organization in plunge-frozen, rehydrated, and immunolabeled tobacco pollen tube. a Confocal medial section; b projection. The cortical-actin fringe is readily apparent in the apical domain as revealed in both a confocal medial optical section (a), and a whole cell projection of all the optical sections (b). $\operatorname{Bar}=10 \mu \mathrm{m}$

which we have prior knowledge of their distribution. In this regard, microtubules are of particular interest since previous reports have described a fringe in the apical domain where the microtubules are more densely organized than in the shank of the tube (Derksen et al. 1985; Raudaskoski et al. 1987; Heslop-Harrison and HeslopHarrison 1988; Del Casino et al. 1993; He and Wetzstein 1995; Foissner et al. 2002). Confocal imaging of whole mounts probed with anti-tubulin antibodies reveals a microtubule fringe (Fig. 5a), which is located at the base of the clear zone and thus behind the actin fringe. As with actin, there are numerous longitudinally oriented microtubules in the shank, but these are much less densely disposed than in the fringe. A few microtubules also appear to create a funnel, but mainly they reside in the cortex. We further note that the microtubule fringe always locates to the base of the clear zone, never within it, and thus occupies a domain further from the apex than the actin fringe. Overall, the microtubule staining pattern appears similar to that obtained previously with ambient-temperature chemical fixation (e.g. He and Wetzstein 1995; Foissner et al. 2002).

As a further validation of the whole-mount immunofluorescence technique, we labeled lily pollen tubes with antibodies to lily villin. In previous studies, these antibodies were reported to colocalize with actin microfilaments at the light and electron microscopic level (Yokota et al. 1998; Vidali et al. 1999). Although colocalization of villin and actin were shown (Yokota et al. 1998), actin preservation was not optimal, and therefore the structural

Fig. 4 Actin immunolabeling of cryofixed, embedded, and sectioned lily pollen tubes. Following embedment in BMM, the pollen tubes were sectioned ( $2 \mu \mathrm{m}$ thick), deresinated, and probed with an anti-actin antibody. a Confocal micrograph of a roughly medial section (note the similarity to whole-mount optical sections in Fig. 1f $-\mathrm{j}$ ); b a section closer to the tube edge than a. Confocal microscopy reveals an intense actin labeling in the cortical region of the apex $(\mathbf{a}, \mathbf{b}) . \mathrm{Bar}=10 \mu \mathrm{m}$

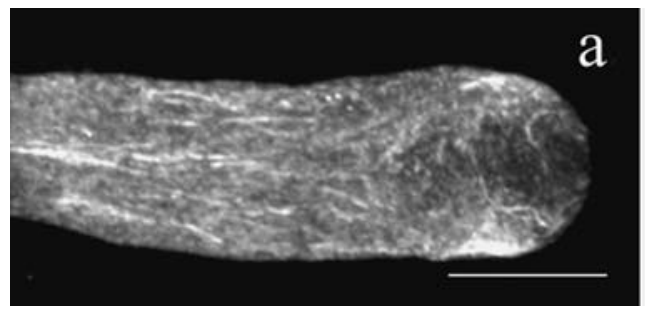

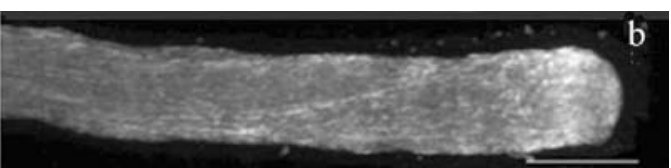

information was not complete. Here we find that villin localizes in the same region as the actin fringe and forms longitudinal filaments throughout the shank of the tube (Fig. 5d). However, there is more diffuse labeling than with anti-actin, perhaps because some of the villin is soluble, and not bound to actin filaments.

An improved ambient-temperature chemical fixation procedure reliably reveals the actin fringe

Although rapid freeze fixation allows us to label the actin cytoskeleton reproducibly, the method is technically demanding; it would be worthwhile to have a viable method for conventional chemical fixation. We were encouraged to think this might be possible because images with resemblance to the ones above have occasionally been published (see Introduction). We began with the simultaneous fixing/staining procedure developed by Foissner et al. (2002). Although we were able to achieve satisfactory fixation on very young pollen tubes and to see the fringe (Fig. 6a-c), this succeeded rarely and for older tubes almost never.

Figure $6 \mathrm{a}-\mathrm{c}$ shows an image of a well-preserved young L. longiflorum pollen tube fixed by this protocol. Unfortunately, it was but one reasonably well-fixed pollen tube in a field of a hundred or more poorly fixed ones. Older tubes were never well preserved, even though we were careful to remove the medium completely before adding the fixative as specified by Foissner et al. (2002). We also applied this method to L. formosanum but had poor results. To enhance preservation, we modified the protocol by replacing EGTA in the fixative with $\mathrm{CaCl}_{2}$, which preserves membrane integrity (Baskin et al. 1992), and by alkalinizing the fixative to $\mathrm{pH} \mathrm{9,} \mathrm{which} \mathrm{accelerates} \mathrm{form-}$ aldehyde cross-linking (Berod et al. 1981). The modified protocol gave images similar to the original without a clear fringe (Fig. 6d-f); but, notably, led to consistent staining among individuals, in both young and old pollen tubes. An additional problem is that the cytoplasm in the chemically fixed cells, in contrast to those prepared by cryofixation, becomes coagulated, a condition that appears to exacerbate over time (Fig. 6f).

A major improvement was made with the addition of $5 \mathrm{mM}$ ethylene glycol bis[succinimidylsuccinate] (sulfoEGS) (Abdella et al. 1979) to the fixation buffer, as de-

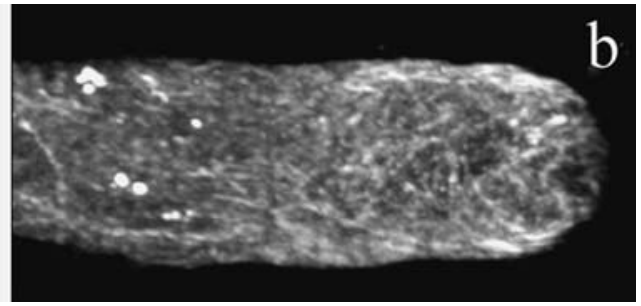



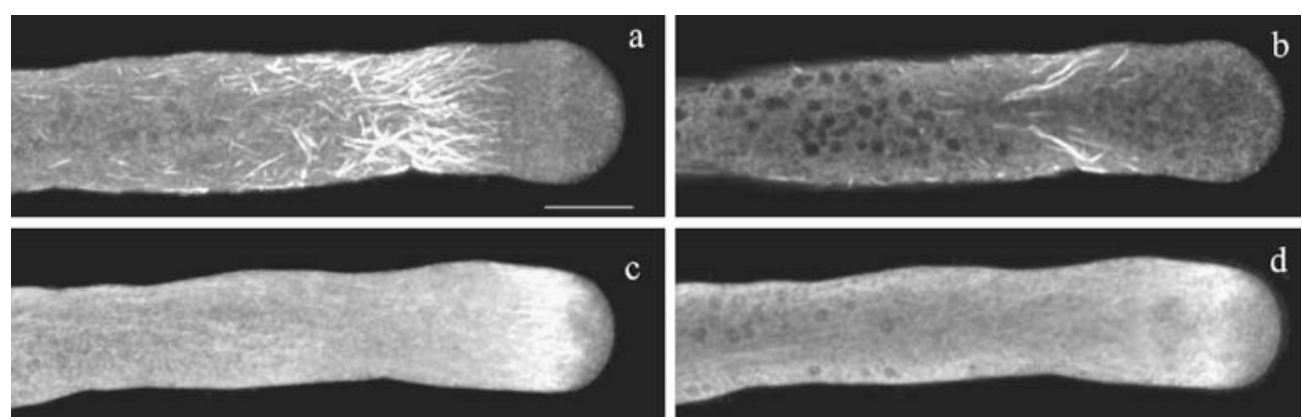

Fig. 5 Microtubule and villin organization in plunge frozen wholemounts of lily pollen tubes. a, b Microtubule staining; a confocal projection image, b medial optical section. Microtubules form a distinct fringe at the base of the clear zone $15 \mu \mathrm{m}$ from the apex. The mid-plane optical section b shows microtubules attaching at the cortex, but most elements curve inwards to the core of the pollen tube. c, d Villin staining; c confocal projection image; d medial optical section. Villin labels the actin fringe, where the signal appears filamentous. This probe can also appear diffuse, as shown in the projection of confocal optical sections (c), and the single confocal medial optical section (d). Bar $=10 \mu \mathrm{m}$

scribed above. With this reagent, the cortical actin fringe (Fig. 7; right panels) is preserved in over 90\% (265/280) of the lily pollen tubes.

Sulfo-EGS (Abdella et al. 1979) is chemically similar to maleimidobenzoyl hydroxysuccinimide (MBS), which was widely used to stabilize actin in plant cells (Sonobe and Shibaoka 1989; Vidali et al. 2001). However, when compared to sulfo-EGS, we find that MBS, gives rise to coarser filaments and more coagulation in the clear zone (results not shown), although the fringe is more reliably preserved than without MBS (Fig. 7k, 1). As with cryofixation, in ambient temperature fixation with sulfo-

Fig. 6 Pollen tubes chemically fixed at ambient temperature and stained with phalloidin. a, c Images of a young L. longiflorum pollen tube fixed by the Foissner et al. (2002) method; a confocal projection image, b medial optical section, c transmitted light image. The images of the apical region do not show the actin fringe clearly. d-f Images of a $L$. formosanum pollen tube fixed by our partially optimized method (i.e., no EGTA, $0.5 \mathrm{mM} \mathrm{CaCl}_{2}, \mathrm{pH} 9$ ). The actin fringe and clear zone are preserved partially; $\mathbf{d}$ projection image; e medial optical section; f DIC. Bar $=10 \mu \mathrm{m}$

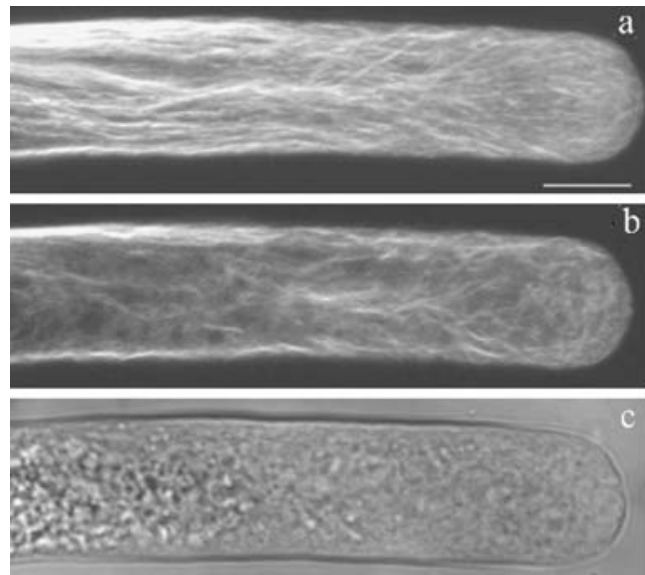

EGS, the fine structure of the actin filaments closely resembles that seen in cryofixed whole-mounts, including the size and structure of the fringe, its variable position from the extreme apex (sometimes forming a caplike structure as in Fig. 7j), the linking of cortex and interior by a few prominent bundles, and evenly dispersed actin bundles throughout the shank. The latter is particularly noteworthy because in conventionally fixed pollen tubes as well as those studied here with MBS replacing the sulfo-EGS, actin filaments in the shank are widely observed to be clumped into a few thick cables.

To determine whether a high $\mathrm{pH}$ is necessary, ambient-temperature fixation was performed at $\mathrm{pH} 7$, with $0.5 \mathrm{mM} \mathrm{CaCl}$, and $5 \mathrm{mM}$ sulfo-EGS; however, the frequency with which the actin fringe was preserved was much lower than at $\mathrm{pH} 9$ (results not shown). Because pH 9 is beyond the buffering range of PIPES, we used instead $100 \mathrm{mM}$ TAPS, which is an effective buffer at this alkaline $\mathrm{pH}$. This change did not improve the results, suggesting that buffering per se is not required. There is also evidence from electron microscopic studies that PIPES, for reasons that have not been resolved, is better than other buffers for fixation (Salema and Brandao 1973). When fixing with sulfo-EGS, and PIPES buffer at $\mathrm{pH} 9$, the addition of supplemental calcium did not influence the preservation of the actin fringe.

Based on the actin structure in plunge frozen, rehydrated, and immunolabeled whole mounts, we have drawn a schematic diagram of actin organization in lily pollen tubes (Fig. 8). The actin fringe, packed with longitudinally oriented actin bundles, is the dominant feature in the subapical region of the pollen tube, as

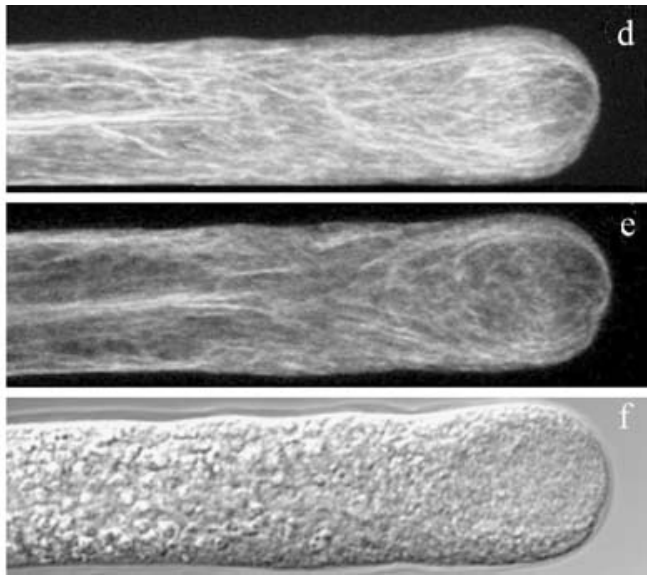


Fig. 7 Images of lily pollen tubes fixed at room temperature with succimidylsuccinate esters (sulfo-EGS or MBS) and labeled with rhodaminephalloidin. Left panels show medial confocal optical sections; the images in the right panel are projections. The first five rows $(\mathbf{a}-\mathbf{j})$ show pollen tubes fixed with sulfo-EGS in the optimized fixation buffer (i.e., $0.5 \mathrm{mM} \mathrm{CaCl}_{2}, \mathrm{pH}$ 9). Note the intensely labeled actin fringe. The last row $(\mathbf{k}, \mathbf{l})$ shows a pollen tube fixed with MBS replacing the sulfo-EGS. Note the bundling of actin filaments in the shank. Bar $=10 \mu \mathrm{m}$
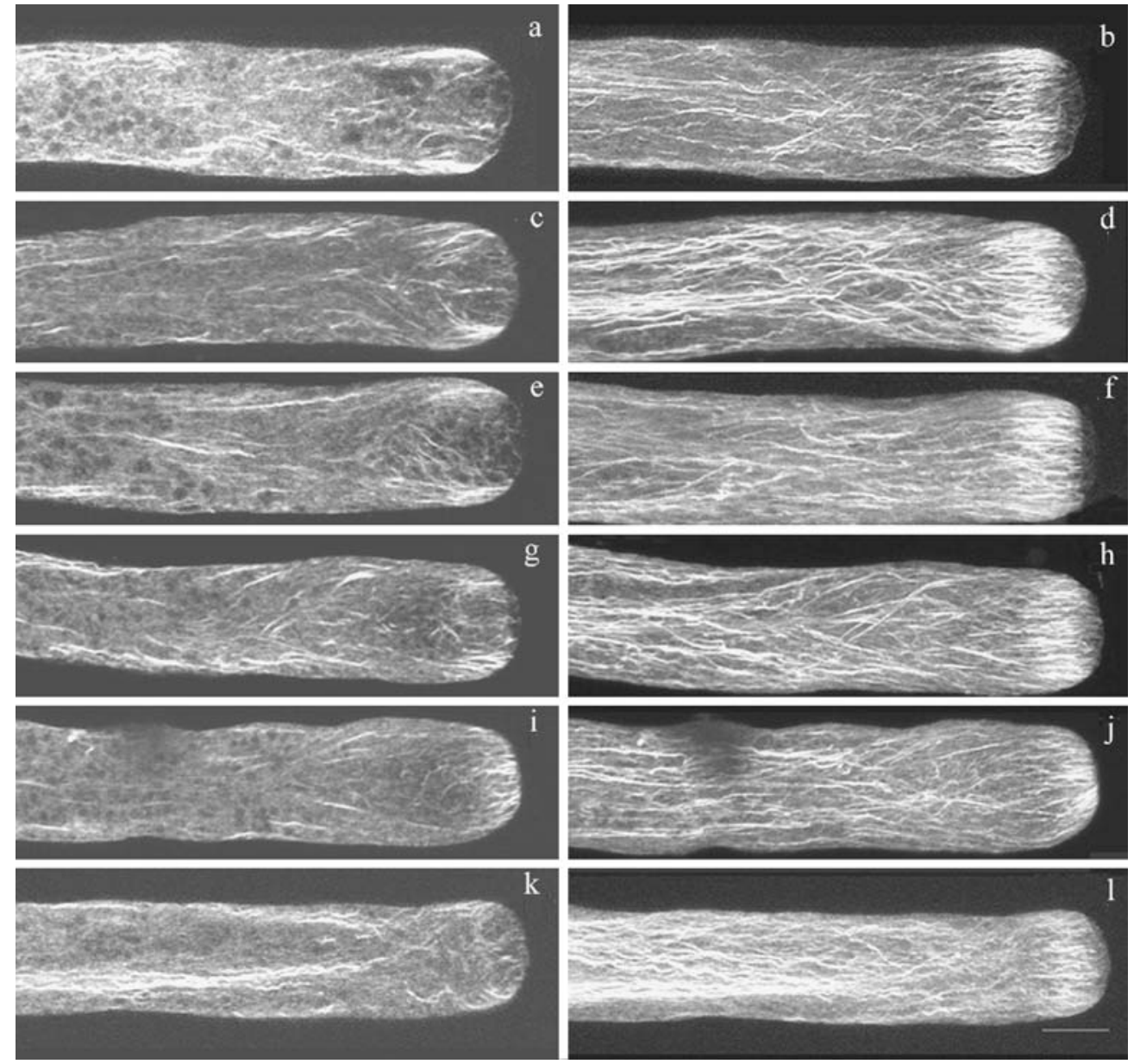

shown in the top panel. The arrow shows that the position of the fringe changes, most likely reflecting different stages of growth oscillations. The bottom right panel shows a pollen tube viewed on end, and illustrates that this actin fringe is largely confined to the cell cortex. The shank of the pollen tube is filled with long actin microfilaments, which are evenly distributed throughout the volume of the cell. We conclude that an apical cortical fringe and fine bundles dispersed through the shank characterize actin organization in the pollen tube.

\section{Discussion}

Our results demonstrate that the apical domain of in vitro grown lily pollen tubes contains a prominent cortical fringe or collar of actin microfilaments, and that the shank possesses an evenly dispersed array of fine actin bundles. The consistency of these results rests on the successful optimization of two distinct structural approaches. The first involves rapid freeze fixation followed by freeze substitution, while the second uses reagents at room temperature to achieve the stabilization of actin microfilaments. Given the well-known ability of cryofixation to faithfully preserve cell struc- ture, we think that this method provides the most reliable way to fix F-actin.

Plant cells are usually not permeable to antibodies unless the cell wall is breached, either with digesting enzymes (Wick et al. 1981; Miller et al. 1995; Vidali and Hepler 1997), or by mechanical force (Wasteneys et al. 1997). Freezing fungal hyphae in sub-optimal conditions, which causes formation of large ice crystals, has also been reported to allow antibody permeation (Bourett et al. 1998). In the current study, cell-wall digestion was not required; apparently, cryofixation produced fissures in the pollen tube cell wall that permitted the entry of antibodies. As with lily, tobacco pollen tubes exhibited a fringe. However, applying the cryofixation and whole-mount staining procedure to tobacco pollen tubes is more difficult than to lily. The clear zone in tobacco is significantly shorter than in lily, making assessment of pollen tube health more uncertain. Another source of the problem may be the cell wall; because it differs compositionally from lily (Li et al. 1994), the tobacco pollen tube may be less prone to disruption during the freeze-substitution procedure and thus less permeable than the lily pollen tube to antibodies.

The improved room-temperature chemical fixation procedure has also faithfully preserved the apical actin 


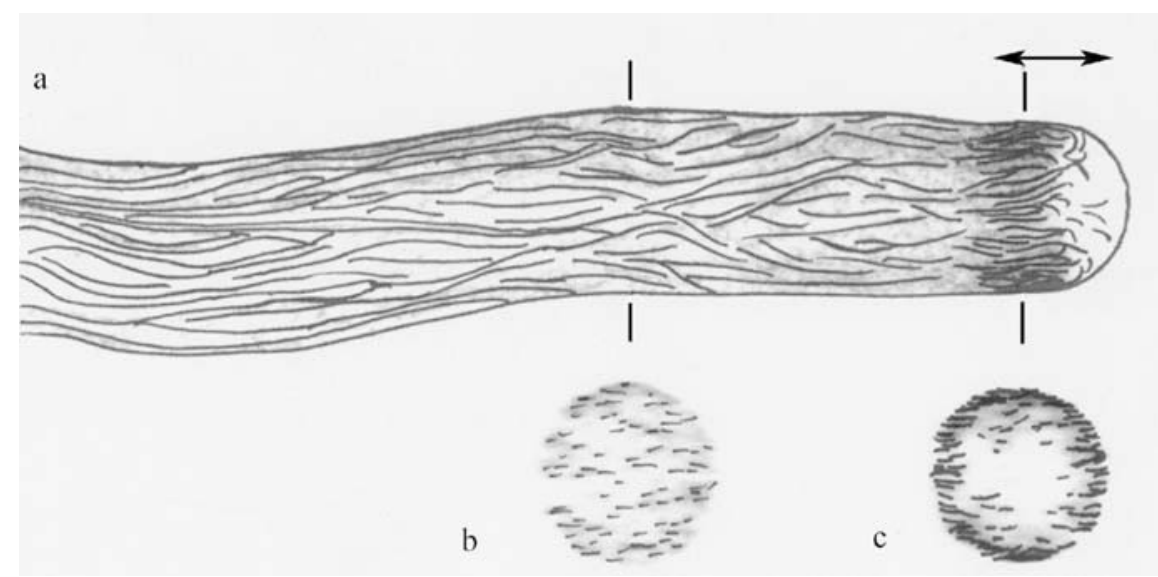

Fig. 8 Schematic image of the organization of actin microfilaments in lily pollen tubes. This model is based on plunge-frozen, immunolabeled, pollen tube whole mounts, and was constructed by tracing the prominent actin filaments in Fig. 2a. The top panel (a) is a longitudinal view; it illustrates a dense actin fringe located 1-5 $\mu \mathrm{m}$ from the apex, and fine actin microfilaments throughout the shank. The double-sided arrow shows that the position of the fringe varies, perhaps depending on the phase of the growth oscillation during which the tube was fixed. The bottom panels $(\mathbf{b}, \mathbf{c})$ show two transverse views. One view (b) bisects the shank of the tube, approximately $40 \mu \mathrm{m}$ behind the apex, and reveals that the actin microfilaments are spread evenly throughout the thickness of the pollen tube, and are not concentrated in any particular region. The other view (c) bisects the fringe, approximately $10 \mu \mathrm{m}$ behind the apex, and indicates that nearly all the actin microfilaments are located in the cell cortex

fringe and the dispersed shank microfilaments, providing a method that is easier to use than cryofixation. Our protocol introduces several novel features. One of these, the use of a succidimidyl ester cross-linker, sulfo-EGS (Abdella et al. 1979), follows closely on previous work with MBS, a related molecule (Sonobe and Shibaoka 1989). Apparently, subjecting the cell to a combination of cross-linking reagents is able to preserve fragile actin microfilaments. In this regard, a recent report demonstrates that using formaldehyde in combination with methylglyoxal, a cross-linking compound analogous to sulfo-EGS, allows high quality images of actin in fungal hyphae (Yu et al. 2004).

The other two changes introduced here, i.e., fixing in the presence of calcium and at $\mathrm{pH} 9$, may seem counterintuitive, particularly in view of the widespread use of buffers at $\mathrm{pH} 7$ with EGTA. It seems likely that the damage to membranes resulting from the removal of calcium, is more detrimental to cell structure than is calcium-induced depolymerization of F-actin or microtubules in the presence of aldehyde fixatives (Baskin et al. 1992). Regarding elevated $\mathrm{pH}$, it is reported that this condition favors cross-linking by formaldehyde (Berod et al. 1981; Eldred et al. 1983), thus permitting a more rapid stabilization of cellular structure than can be achieved at lower $\mathrm{pH}$. It is well known from the studies of pollen tubes that slow fixation leads to structural artifacts (Doris and Steer 1996); thus, the ability to hasten the cross-linking process at $\mathrm{pH} 9$ may be important to preserve structures that would otherwise be lost.

The most striking aspect of actin organization revealed by our new methods is the detail and consistency of the apical fringe, which is composed of longitudinal microfilaments localized in the cell cortex. The individual cortical filaments extend towards the extreme apex, but stop about $1-5 \mu \mathrm{m}$ from the tip, creating an actin depleted zone at the tip itself. In the tube interior, a few actin filaments often extend all the way to the apex; nevertheless when compared to other regions of the cell the apical most domain has a very low density of actin microfilaments. The presence of an apical zone mostly depleted of actin fits well with the position of the calcium gradient (Holdaway-Clarke and Hepler 2003) and the view that microfilaments in this domain would be degraded by the actin-binding protein villin, or gelsolin. Villin was shown to unbundle actin in the presence of calcium and calmodulin (Yokota et al. 1998), whereas a gelsolin-like protein, which has recently been identified in poppy pollen, was implicated in the fragmentation of actin filaments (Huang et al. 2004).

We recognize that an apical actin fringe has previously been reported (Pierson et al. 1986; Yokota et al. 1995; Kost et al. 1998; Gibbon et al. 1999). In a review, Geitmann and Emons (2000), have even diagramed a pollen tube with a fringe, which they based on previous studies of angiosperm pollen tubes, as well as growing root hairs, and moss protonemata. Despite these few positive examples, it is important to emphasize that the fringe is not consistently observed. Indeed, in a recent report Foissner et al. (2002) claim that the apical domain of lily pollen tubes is characterized by a random network of fine filaments, although curiously they see a fringe in pollen tubes that were treated with the phosphatase inhibitor, calyculin A. In contrast to these reports, we find here, with both our procedures that the fringe is present in virtually every pollen tube. Those few examples where we do not see a fringe can be excluded due to salient problems with the pollen tube itself, such as an abnormal morphology or a short length, indicating poor growth, or poor penetration of the antibody. Therefore, 
we argue that the cortical fringe is a fundamental attribute of the actin cytoskeleton in the growing pollen tube.

Our attempt to develop a consensus about the structure of microfilaments in the apical domain of the growing pollen tube is ultimately aimed at constructing a model that will help us explain how actin contributes to pollen tube growth. In previous efforts, for example, Mathur and Hülskamp (2001) model the apical domain of tip-growing cells as a branching network of actin, very similar to the way these elements are portrayed in lamellipodia of migrating animal cells (Pollard 2003). Given previous reports of random arrays of actin in the apical domain (Fu et al. 2001; Foissner et al. 2002), this is a reasonable model. However, with the present results, we feel that this representation of the actin structural organization and its associated model can be rejected, at least for the pollen tube.

The inability of standard methods to preserve the actin fringe indicates that it is a fragile structure, in which the microfilaments may not be tightly cross-linked, and thus labile. It also seems likely that it represents a rapidly turning over array of actin. In this regard, we note that of the live cell probes, GFP-ADF best identifies the fringe (Chen et al. 2002, K. Wilsen, unpublished observation). $\mathrm{ADF}$ is well known to participate in the remodeling of actin, especially in regions of elevated $\mathrm{pH}$. Because both ADF (Chen et al. 2002) and the fringe are located in a region of the clear zone that is alkaline (Feijó et al. 1999), the conditions are present that would support active remodeling of the actin cytoskeleton. An additional important feature of the fringe is its localization in the cortex, close to the apical plasma membrane, where phosphatidyl inositol 4,5 bisphosphate is specifically known to reside (Kost et al. 1999). Because phosphatidyl inositol 4,5 bisphosphate associates with different actinbinding proteins, including notably $\mathrm{ADF}$, villin and profilin (Martin 1998), several factors are present that could contribute to the nucleation, and growth of new actin microfilaments. Since the fringe must keep pace with growth, or even anticipate the process, and since average rates of $0.3 \mu \mathrm{m} / \mathrm{s}$ occur in lily, this would mean that a $5-10 \mu \mathrm{m}$ wide fringe would completely turnover every $16-33 \mathrm{~s}$. In addition to polymerization, we must also consider that the cortical fringe acts as a track along which myosin can transport vesicles to precise sites in the apex of the pollen tube, and in this way contribute to polarized growth.

Besides the actin fringe, confocal images of cells labeled with antibodies to tubulin indicate that there is a microtubule fringe located at the base of the clear zone. The presence of a microtubule fringe, although not seen is all previous studies, is more readily apparent in conventionally fixed cells than the actin fringe. Presumably microtubules are more stable than actin microfilaments. However, they too must turn over at a rate consonant with growth. Surprisingly, oryzalin $(0.5 \mu \mathrm{M})$, a potent microtubule depolymerizing drug, has no effect on tobacco pollen tube growth (Aström et al. 1995), and colchicine $(25 \mu \mathrm{M})$ and propham $(70 \mathrm{nM})$ cause only a
$20 \%$ inhibition (Joos et al. 1994). These drugs do, however, inhibit the motility of the male germ unit, and lead to cytoplasmic disorganization, with vacuoles moving towards the tip of the tube, suggesting that microtubules are involved in these intracellular motility processes (Heslop-Harrison and Heslop-Harrison 1988; Joos et al. 1994; Aström et al. 1995).

In conclusion, the use of rapidly frozen whole mounts as well as an improved room temperature fixation method have revealed the apical actin fringe located in the cell cortex as a constant feature of growing pollen tubes. These observations may help provide clarity with regard to the confusion surrounding the structure of actin in these rapidly growing cells. Additionally, these methods may be useful for studies on other plant and animal cells, in which there is confusion about the structure and organization of the actin cytoskeleton.

Acknowledgements We thank Dale Callaham for his excellent technical assistance, and acknowledge the National Science Foundation grant that supports the Central Microscopy Facility (NSF BBS 8714235), where all images were acquired. We thank Lawrence Hurd for suggesting the use of a high $\mathrm{pH}$ during ambient-temperature fixation. We also thank Dr. Kent McDonald, University of California, Berkeley, CA for helpful discussions about cryofixation strategies and together with Dr. J. Sedat, University of California, San Francisco, CA for drawing our attention to EGS as a potential cross-linker for actin fixation. The Davis and Delisle Funds, of the Plant Biology Graduate Program, are acknowledged for their support. We thank the Gloeckner Company for supplying us with L. longiflorum bulbs. This project was supported by the National Science Foundation grant No. MCB-0077599 to PKH, and U.S. Department of Energy grant No. 03ER15421 to TIB, which does not constitute endorsement by the Department of views expressed herein.

\section{References}

Abdella RM, Smith PK, Royer GP (1979) A new cleavable reagent for crosslinking and reversible immobilization of proteins. Biochem Biophys Res Commun 87:734-742

Aström H, Sorri O, Raudaskoski M (1995) Role of microtubules in the movement of the vegetative nucleus and generative cell in tobacco pollen tubes. Sex Plant Reprod 8:61-69

Baskin TI, Busby CH, Fowke LC, Sammut M, Gubler F (1992) Improvements in immunostaining samples embedded in methacrylate: localization of microtubules and other antigens throughout developing organs in plants of diverse taxa. Planta 187:405-413

Baskin TI, Miller DD, Vos JW, Wilson JE, Hepler PK (1996) Cryofixing single cells and multicellular specimens enhances structure and immunocytochemistry for light microscopy. J Microsc 182:149-161

Berod A, Hartman BK, Pujol JF (1981) Importance of Fixation in Immunohistochemistry-use of formaldehyde solutions at variable ph for the localization of tyrosine-hydroxylase. J Histochem Cytochem 29:844-850

Bourett TM, Czymmek KJ, Howard RJ (1998) An improved method for affinity probe localization in whole cells of filamentous fungi. Fungal Genet Biol 24:3-13

Chen CY, Wong EI, Vidali L, Estavillo A, Hepler PK, Wu HM, Cheung AY (2002) The regulation of actin organization by actin-depolymerizing factor in elongating pollen tubes. Plant Cell 14:2175-2190

Condeelis JS (1974) Identification of F-Actin in pollen tube and protoplast of Amaryllis belladona. Exp Cell Res 88:435-439 
Del Casino C, Li Y-Q, Moscatelli A, Scali M, Tiezzi A, Cresti M (1993) Distribution of microtubules during the growth of tobacco pollen tubes. Biol Cell 79:125-132

Derksen J, Pierson ES, Traas JA (1985) Microtubules in vegetative and generative cells of pollen tubes. Eur J Cell Biol 38:142-148

Derksen J, Rutten T, van Amstel T, Dewin A, Doris F, Steer M (1995) Regulation of pollen tube growth. Acta Botanica Neerl 44:93-119

Doris FP, Steer MW (1996) Effects of fixatives and permeabilisation buffers on pollen tubes: implications for localisation of actin microfilaments using phalloidin staining. Protoplasma 195:25-36

Eldred W, Zucker C, Karten H, Yazulla S (1983) Comparison of fixation and penetration enhancement techniques for use in ultrastructural immunocytochemistry. J Histochem Cytochem 31:285-292

Fields SD, Strout GW, Russell SD (1997) Spray-freezing freeze substitution (SFFS) of cell suspensions for improved preservation of ultra structure. Microsc Res Tech 38:315-328

Feijó JA, Sainhas J, Hackett GR, Kunkel JG, Hepler PK (1999) Growing pollen tubes possess a constitutive alkaline band in the clear zone and a growth-dependent acidic tip. J Cell Biol 144:483-496

Foissner I, Grolig F, Obermeyer G (2002) Reversible protein phosphorylation regulates the dynamic organization of the pollen tube cytoskeleton: effects of calyculin A and okadaic acid. Protoplasma 220:1-15

Fu Y, Wu G, Yang Z (2001) Rop GTPase-dependent dynamics of tip-localized f-actin controls tip growth in pollen tubes. J Cell Biol 152:1019-1032

Geitmann A, Emons AM (2000) The cytoskeleton in plant and fungal cell tip growth. J Microsc 198:218-245

Gibbon BC, Kovar DR, Staiger CJ (1999) Latrunculin B has different effects on pollen germination and tube growth. Plant Cell 11:2349-2363

He Y, Wetzstein H (1995) Fixation induces differential tip morphology and immunolocalization of the cytoskeleton in pollen tubes. Physiol Plant 93:757-763

Heslop-Harrison J, Heslop-Harrison Y (1988) Cytoskeletal elements, cell shaping and movement in the angiosperm pollen tube. J Cell Sci 91:49-60

Heslop-Harrison J, Heslop-Harrison Y (1991) The actin cytoskeleton in unfixed pollen tubes following microwave-accelerated DMSO-permeabilisation and TRITC-phalloidin staining. Sex Plant Reprod 4:6-11

Holdaway-Clarke TL, Hepler PK (2003) Control of pollen tube growth: role of ion gradients and fluxes. New Phytol 159:539563

Huang SJ, Blanchoin L, Chaudhry F, Franklin-Tong VE, Staiger CJ (2004) A gelsolin-like protein from Papaver rhoeas pollen (PrABP80) stimulates calcium-regulated severing and depolymerization of actin filaments. J Biol Chem 279:23364-23375

Joos U, van Aken J, Kristen U (1994) Microtubules are involved in maintaining the cellular polarity in pollen tubes of Nicotiana sylvestris. Protoplasma 179:5-15

Kost B, Spielhofer P, Chua NH (1998) A GFP-mouse talin fusion protein labels plant actin filaments in vivo and visualizes the actin cytoskeleton in growing pollen tubes. Plant $\mathbf{J}$ 16:393-401

Lancelle SA, Hepler PK (1989) Immunogold labelling of actin on sections of freeze-substituted plant cells. Protoplasma 150:72-74

Lancelle SA, Hepler PK (1991) Association of actin with cortical microtubules revealed by immunogold localization in Nicotiana pollen tubes. Protoplasma 165:167-172

Lancelle SA, Hepler PK (1992) Ultrastructure of freeze-substituted pollen tubes of Lilium longiflorum. Protoplasma 167:215-230

Lancelle SA, Callaham DA, Hepler PK (1986) A method for rapid freeze fixation of plant cells. Protoplasma 131:153-165

Li YQ, Chen F, Linskens JF, Cresti M (1994) Distribution of unesterified and esterified pectins in cell walls of pollen tubes of flowering plants. Sex Plant Reprod 7:145-152

Li Y, Zee SY, Liu YM, Huang BQ, Yen LF (2001) Circular F-actin bundles and a G-actin gradient in pollen and pollen tubes of Lilium davidii. Planta 213:722-730
Martin TFJ (1998) Phosphoinositide lipids as signaling molecules: common themes for signal transduction, cytoskeletal regulation, and membrane trafficking. Annu Rev Cell Dev Biol $14: 231-264$

Mathur J, Hülskamp M (2001) Cell growth: how to grow and where to grow. Curr Biol 11:R402-R404

Miller DD, Scordilis SP, Hepler PK (1995) Identification and localization of three classes of myosins in pollen tubes of Lilium longiflorum and Nicotiana alata. J Cell Sci 108:2549-2563

Miller DD, Lancelle SA, Hepler PK (1996) Actin microfilaments do not form a dense meshwork in Lilium longiflorum pollen tube tips. Protoplasma 195:123-132

Perdue TD, Parthasarathy MV (1985) In situ localization of Factin in pollen tubes. Eur J Cell Biol 39:13-20

Pierson ES (1988) Rhodamine-phalloidin staining of F-actin in pollen after dimethylsulphoxide permeabilization. Sex Plant Reprod 1:83-87

Pierson ES, Derksen J, Traas JA (1986) Organization of microfilaments and microtubules in pollen tubes grown in vitro or in vivo in various angiosperms. Eur J Cell Biol 41:14-18

Pollard TD (2003) The cytoskeleton, cellular motility and the reductionist agenda. Nature 422:741-745

Raudaskoski M, Aström H, Perttila K, Virtanen I, Louhelainen J (1987) Role of the microtubule cytoskeleton in pollen tubes: an immunocytochemical and ultrastructural approach. Biol Cell 61:177-188

Raudaskoski M, Aström H, Laitiainen E (2001) Pollen tube cytoskeleton: structure and function. J Plant Growth Regul 20:113130

Salema R, Brandao I (1973) The use of PIPES buffer in the fixation of plant cells for electron microscopy. J Submicr Cytol 5:79-96

Sonobe S, Shibaoka H (1989) Cortical fine actin filaments in higher plant cells visualized by rhodamine-phalloidin after pretreatment with m-maleimidobenzoyl N-hydroxysuccinimide ester. Protoplasma 148:80-86

Tang X, Lancelle SA, Hepler PK (1989) Fluorescence microscopic localization of actin in pollen tubes: comparison of actin antibody and phalloidin staining. Cell Motil Cytoskel 12:216224

Tiwari SC, Polito VS (1988) Organization of the cytoskeleton in pollen tubes of Pyrus communis: a study employing conventional and freeze-substitution electron microscopy, immunofluorescence, and rhodamine-phalloidin. Protoplasma 147:100 112

Vidali L, Hepler PK (1997) Characterization and localization of profilin in pollen grains and tubes of Lilium longiflorum. Cell Motil Cytoskeleton 36:323-338

Vidali L, Yokota E, Cheung AY, Shimmen T, Hepler PK (1999) The $135 \mathrm{kDa}$ actin-bundling protein from Lilium longiflorum pollen is the plant homologue of villin. Protoplasma 209:283291

Vidali L, McKenna ST, Hepler PK (2001) Actin polymerization is necessary for pollen tube growth. Mol Biol Cell 12:2534-2545

Wasteneys GO, Willingale-Theune J, Menzel D (1997) Freeze shattering: a simple and effective method for permeabilizing higher plant cell walls. J Microsc-Oxford 188:51-61

Wick SM, Seagull RW, Osborn M, Weber K, Gunning BES (1981) Immunofluorescence microscopy of organized microtubule arrays in structurally stabilized meristematic plant cells. J Cell Biol 89:685-690

Yokota E, McDonald AR, Liu B, Shimmen T, Palevitz BA (1995) Localization of a $170 \mathrm{kDa}$ myosin heavy chain in plant cells. Protoplasma 185:178-187

Yokota E, Takahara K, Shimmen T (1998) Actin-bundling protein isolated from pollen tubes of lily: biochemical and immunocytochemical characterization. Plant Physiol 116:1421-1429

Yu YP, Jackson SL, Garrill A (2004) Two distinct distributions of F-actin are present in the hyphal apex of the oomycete Achlya bisexualis. Plant Cell Physiol 45:275-280 